\title{
A Mathematical Model for the Assessment of Hemodynamic Parameters Using Quantitative Contrast Echocardiography
}

\author{
Xucai Chen,* Karl Q. Schwarz, Daniel Phillips, Sherry D. Steinmetz, and Reinhard Schlief
}

\begin{abstract}
A mathematical model for the assessment of hemodynamic parameters using quantitative echocardiography is presented. The method involves the intravenous injection of an ultrasonic echo contrast agent. The relative enhancement of the backscattered ultrasound intensity is measured as a function of time (the time-intensity curve). From this measurement, the volume flow rate (cardiac output) and the mixing volume are calculated. Relevant acoustic properties of the ultrasound contrast agent are discussed. An in vitro experiment is performed to corroborate the theory presented.
\end{abstract}

Index Terms - Attenuation, contrast agent, hemodynamics, scattering.

\section{INTRODUCTION}

$\mathbf{H}$ EMODYNAMIC parameters such as the cardiac output are important to the diagnosis of many disease states. A variety of techniques are available, including the Fick method, indicator dilution techniques, velocity measurements and transthoracic impedance, and Doppler ultrasound techniques. A review of these techniques is given by Ehlers et al. [1]. Among the indicator dilution techniques, thermal dilution and bolus injection of indocyanine green have had reasonable success. Ultrasound imaging techniques are, in general, noninvasive. With the development of bubble-based ultrasound contrast agents, it is theoretically possible to institute a noninvasive technique to measure cardiac hemodynamic parameters. Some earlier attempts made are summarized by Feinstein et al. [2]

Classical indicator dilution theory is based on the law of mass conservation. After a dose of a suitable indicator (the agent) is injected into the patient's blood, the concentration of the agent is monitored as a function of time. The area under the concentration curve is relatively insensitive to the time history

Manuscript received November 19, 1996; revised December 3, 1997. This work was supported in part by the American Heart Association, New York State Affiliate, Inc. under a Grant-In-Aid. Asterisk indicates corresponding author.

*X. Chen is with the Department of Medicine, Box 679 , University of Rochester, Rochester, NY 14642 USA (e-mail: xucai_chen@urmc.rochester.edu).

K. Q. Schwartz and S. D. Steinmetz are with the Department of Medicine and Rochester Center for Biomedical Ultrasound, University of Rochester, Rochester, NY 14642 USA.

D. Phillips is with the Department of Electrical Engineering and Rochester Center for Biomedical Ultrasound, University of Rochester, Rochester, NY 14642 USA.

R. Schlief is with the Department of Clinical Development Magnetic Resonance and Ultrasound Contrast Media, Schering, AG, Berlin, Germany.

Publisher Item Identifier S 0018-9294(98)03716-1. of the injection, as long as the total dose is delivered over a relatively short period of time. The peak effect, however, does vary considerably. The means to monitor the concentration of the agent in the blood depends on the particular property of the agent. For example, optical absorption is used to monitor the concentration of the indocyanine green dye (ICGD).

The contrast enhancement effect of an ultrasound contrast agent can be utilized in many ways. Current clinical indications for contrast echocardiography are improved boundary definition and enhanced flow visualization using two-dimensional imaging and Doppler techniques. When used in this way, the sonographer benefits from a prolonged, steady-state contrast effect. The duration of contrast effect (length of time in which the detected signal is above background by a threshold number, such at $3 \mathrm{~dB}$ ) is expected to be proportional to the logarithm of the dose divided by the total blood volume [3]. When administered at the recommended (pharmacological) dose, there is an initial shadowing effect during a short time subsequent to the initial injection, commonly referred to as the first pass of the agent, followed by a long period of decreasing enhancement. The initial shadowing is due to the attenuation properties inherent in the contrast agent. It does not affect the overall performance of the agent for contrast enhancement. However, if the primary goal of utilizing an injection of a contrast enhancement agent is the quantitative indicator-dilution style measurements of cardiac parameters, the first pass parameters must be measured accurately. The duration of contrast effect is actually of less importance in this case. Instead, one should strive to obtain accurately the initial part of the intensity-time curve, which can be mathematically related to the actual contrast concentration curve. The effect of attenuation due to the contrast agent plays an important role in the backscatter measurement during the initial segment of the first pass measurements. This effect must be considered when the measured backscatter intensity is used for a quantitative dilution study. As will be shown later, the attenuation effect can be used to obtain an accurate dilution curve.

A mathematical model is presented relating quantitative echocardiography measurements and the hemodynamic parameters which takes this attenuation effect into account. First, the classical indicator dilution theory is reviewed. In this case, the indicator is the ultrasound contrast enhancement agent. Simple mathematical models are presented to express the concentration of the indicator as a function of time. Next, the backscattered ultrasound signal is related to the concentration 


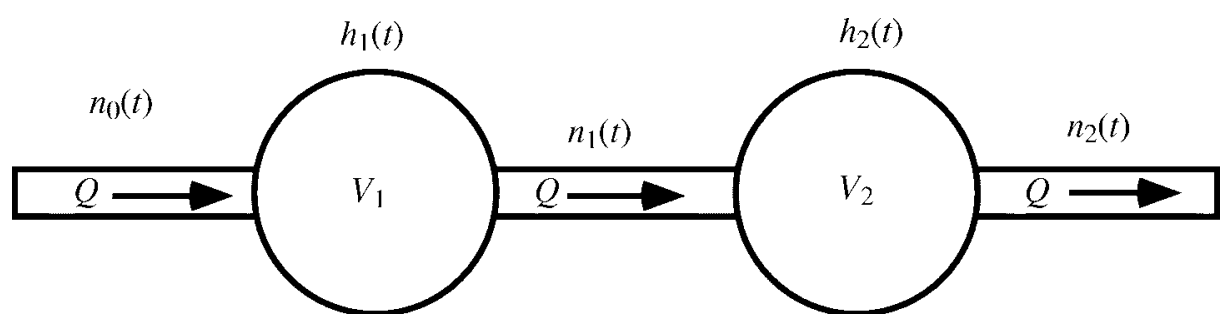

Fig. 1. The flow system is divided into a series of mixing chambers, each of which is described by a transfer function $h_{i}(t)$.

of the indicator, and the effect of attenuation is discussed. Then, an in vitro experimental study is performed to corroborate the theory presented. A tissue phantom is used to simulate the effects of an intravenous injection of ultrasonic echo contrast agent into a flow system. The relative enhancement of the backscattered ultrasound energy is measured as a function of time (the time-intensity curve). From this measurement, the mixing volume and the volume flow rate are calculated. The volume flow rate is also monitored independently. Utilizing this method, system calibration would be performed by using the background scattering from a region of blood in a vessel or soft tissue.

\section{REVIEW OF Dilution THEORY}

After a dose of contrast agent is injected into a vein, it is immediately diluted by the patient's blood. The farther the agent travels in the blood stream, the lower its concentration becomes, at least in the initial segment of the first pass circulation. The contrast agent can be considered completely mixed with the blood pool after many passes through the patient's circulatory system with the concentration of the agent reaching a steady state. Most of the mixing can be considered to occur in a few compartments in the circulatory system, such as the right heart, the lungs, and the left heart. Each of these compartment is treated as a simple mixing chamber, where the mixing process inside the chamber is described by a simple mathematical model.

\section{A. System Model of a Single Mixing Chamber}

The flow system is divided into a series of mixing chambers, as shown in Fig. 1. The volume flow rate in the system is $Q$. The volume of each mixing chamber is $V_{i}$. The mixing volume is sometimes called the volume of distribution of the indicator. The concentration of indicator inside the chamber (the output function) is denoted by $n_{i}(t)$. Regardless of the actual shape of the curves, the time integral under each of these concentration curves must be equal, i.e.,

$$
I=\int_{0}^{\infty} n_{i}(t) d t=D_{0} / Q
$$

where $D_{0}$ is the total amount of agents injected (the dosage). This is governed by the mass conservation law. Equation (1) represents the classical dilution theory.

When the concentration of indicator entering the mixing chamber (the input function) is described as $n_{i-1}(t)$, then concentration of indicator inside the chamber (the output function) is given by

$$
n_{i}(t)=n_{i-1}(t) \otimes h_{i}(t), \quad i=1,2,3, \cdots
$$

where $\otimes$ stands for convolution, and $h_{i}(t)$ is called the transfer function of the mixing chamber. The transfer function can be a complicated function of hemodynamic parameters. The simplest model often used is determined by a single parameter: the time constant of the mixing chamber. This time constant $\tau_{i}$, which can be considered as the system transit time for the mixing chamber, is defined as the ratio of the mixing volume to the volume flow rate, i.e., $\tau_{i}=V_{i} / Q$. The system transfer function for this model is given by

$$
h_{i}(t)= \begin{cases}0, & t<0 \\ \left(1 / \tau_{i}\right) e^{-t / \tau_{i}}, & t \geq 0 .\end{cases}
$$

A delay time related to the travel time of the indicator from the previous mixing chamber to the current mixing chamber (or the observation site) may be incorporated into the transfer function, but it is omitted here for simplicity.

The transfer function given by (3) assumes that the contrast agent is mixed completely with the volume of fluid inside the mixing chamber once the contrast agent enters the chamber. Other transfer functions can be constructed under different assumptions. For example, for perfusion studies, a Gaussian (bell shaped) transfer function can be used.

Other parameters of the concentration-time curve include the centroid defined as

$$
\bar{t}_{i}=\frac{\int_{0}^{\infty} t \cdot n_{i}(t) d t}{\int_{0}^{\infty} n_{i}(t) d t} .
$$

For the simple mixing chamber model given by (3), it can be shown that

$$
\bar{t}_{i}=\bar{t}_{i-1}+\tau_{i}
$$

where $\bar{t}_{i-1}$ is the centroid of the input function, and $\tau_{i}$ is the time constant of the mixing chamber. The above discussion is valid regardless of the actual shape of the input function.

Let us now consider the output function at the first mixing chamber following the injection site. The ideal bolus injection of a contrast agent is prescribed by the delta function, i.e.,

$$
n_{0}(t)=\left(D_{0} / Q\right) \delta(t) .
$$

For this input function, the output function of the first mixing chamber is given by

$$
n_{1}(t)= \begin{cases}0, & t<0 \\ n_{\max , 1} e^{-t / \tau_{1}}, & t \geq 0\end{cases}
$$



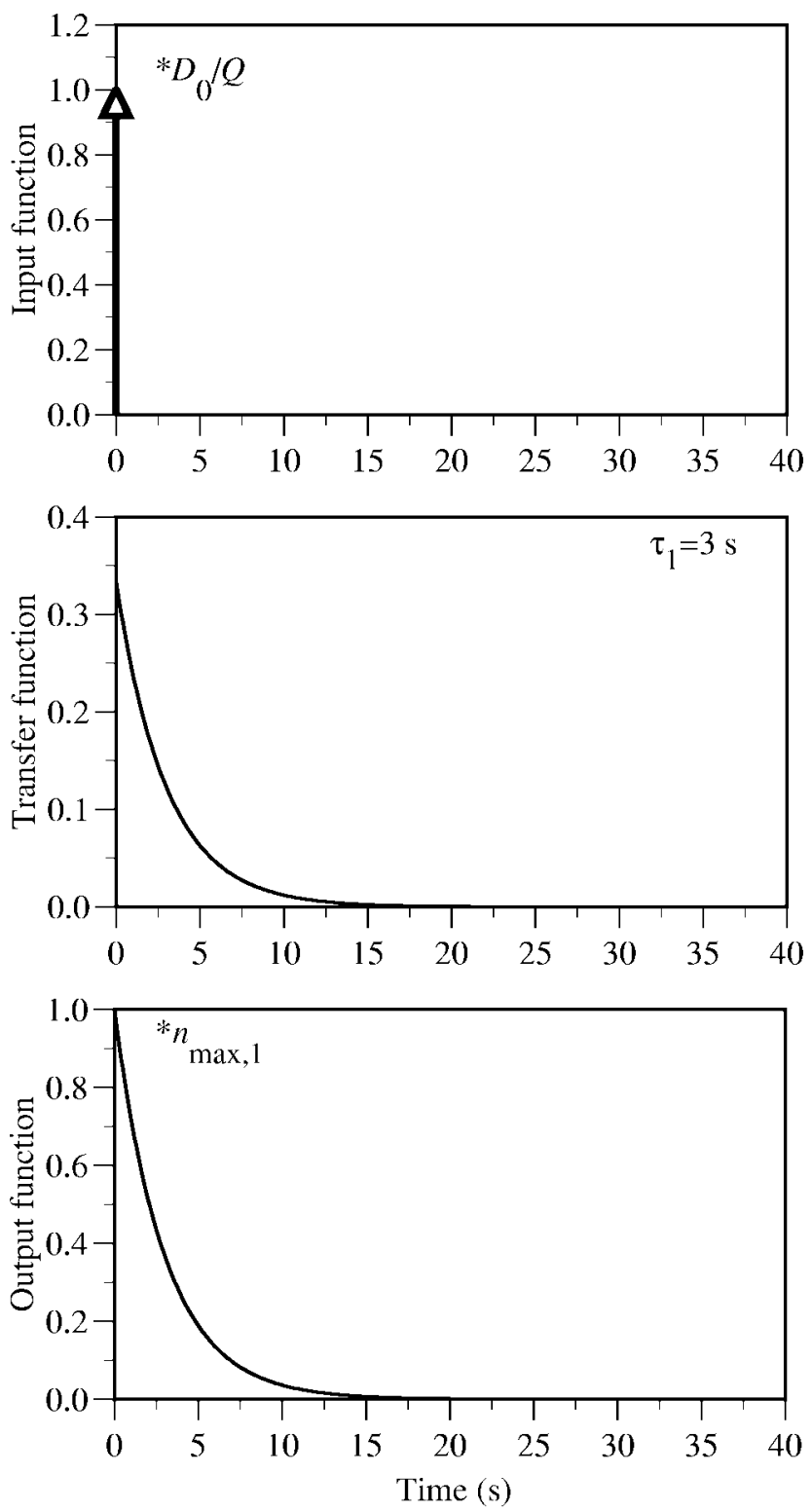

Fig. 2. The dilution curve of a simple mixing chamber after a bolus injection. The input function is given by a delta function. The output function is a simple exponential decay curve.

where $n_{\max , 1}=D_{0} / V_{1}=D_{0} / Q \tau_{1}$ is the peak value of the output function. Fig. 2 shows the behavior of these functions. It is observed immediately that the peak of $n_{1}(t)$ occurs at $t=0$. The concentration curve is described completely by two parameters: $n_{\max , 1}$ and $\tau_{1}$. Once these parameters are determined, we have $Q=D_{0} / n_{\max , 1} \tau_{1}$, and $V_{1}=D_{0} / n_{\max , 1}$. The flow rate and mixing volume can be calculated for such an idealized input function.

In practice, a mixing chamber with such ideal input and output functions is seldom realized. The injected bolus is diluted by a finite volume of fluid before reaching the mixing chamber. In this case, the input function can be approximated by a rectangular function, i.e.,

$$
n_{0}(t)= \begin{cases}D_{0} / V_{0}, & 0<t \leq \tau_{0} \\ 0, & \text { otherwise }\end{cases}
$$

where $V_{0}$ is the volume the contrast is premixed into, and $\tau_{0}=V_{0} / Q$. For such an input function, we have from (2)

$$
n_{1}(t)= \begin{cases}0, & t<0, \\ n_{\max , 1} \frac{1-e^{-t / \tau_{1}}}{1-e^{-\tau_{0} / \tau_{1}},}, & 0 \leq t<\tau_{0} \\ n_{\max , 1} e^{-\left(t-\tau_{0}\right) / \tau_{1}} & t \geq \tau_{0} .\end{cases}
$$

The peak of $n_{1}(t)$ occurs at $t=\tau_{0}$, with a peak value of

$$
\begin{aligned}
n_{\max , 1} & =\frac{D_{0}}{V_{1}} \frac{1-e^{-\tau_{0} / \tau_{1}}}{\tau_{0} / \tau_{1}} \\
& =\frac{D_{0}}{Q} \frac{1-e^{-\tau_{0} / \tau_{1}}}{\tau_{0}} .
\end{aligned}
$$

Fig. 3 shows the behavior of these functions. The concentration curve is described completely by three parameters: $n_{\max , 1}, \tau_{0}$, and $\tau_{1}$. Once these parameters are determined, we have $Q=\left(D_{0} / n_{\max , 1}\right)\left(1-e^{\left.-\tau_{0} / \tau_{1}\right)} / \tau_{0}\right.$, and $V_{1}=$ $\left(D_{0} / n_{\max , 1}\right)\left(1-e^{\left.-\tau_{0} / \tau_{1}\right)} /\left(\tau_{0} / \tau_{1}\right)\right.$. If the premixed volume is much smaller than the mixing volume $\left(V_{0} / V \ll 1\right)$, we have $\tau_{0} / \tau_{1} \ll 1$. In this case, (9) approaches the ideal output function given by (7).

To summarize, when a simple mixing chamber model is assumed, the time constant of the system is equal to the ratio of the mixing volume to the flow rate, while the integral under the concentration-time curve is equal to the ratio of the dose of contrast agent to the volume flow rate. The peak value of the concentration, given by (10), is approximately equal to the dose divided by the mixing volume. In practice, the transfer function of the mixing chamber is not known exactly. However, regardless of the actual shape of the concentration curve, the integral under the concentration curve is equal to the ratio of dose divided by the volume flow rate.

\section{B. Effect of Multiple Mixing Chambers}

When the concentration of the contrast agent is measured after passing through multiple mixing chambers, the concentration inside each chamber can be calculated from (2) by treating the output function from the previous mixing chamber as its input function. Many properties of the dilution curve from a single mixing chamber remain. For example, the integral under the concentration curve for each chamber is equal to the ratio of the dose of the contrast agent used to the volume flow rate, as indicated by (1).

Fig. 4 shows the concentration after two mixing chambers for the rectangular initial input function given by (8).

$$
n_{2}(t)= \begin{cases}0, & t<0 \\
n_{\max , 2}\left\{\begin{array}{ll}
\left.\frac{\tau_{1} e^{\tau_{12} / \tau_{1}}}{\tau_{1}-\tau_{2}} \frac{1-e^{-t / \tau_{1}}}{1-e^{-\tau_{0} / \tau_{1}}}+\frac{\tau_{2} e^{\tau_{12} / \tau_{2}}}{\tau_{2}-\tau_{1}} \frac{1-e^{-t / \tau_{2}}}{1-e^{-\tau_{0} / \tau_{2}}}\right\}, & 0 \leq t<\tau_{0} \\
n_{\max , 2}\left\{\frac{\tau_{1}}{\tau_{1}-\tau_{2}} e^{-\left(t-\tau_{p}\right) / \tau_{1}}+\frac{\tau_{2}}{\tau_{2}-\tau_{1}} e^{-\left(t-\tau_{p}\right) / \tau_{2}}\right.
\end{array}\right\}, & t \geq \tau_{0}\end{cases}
$$



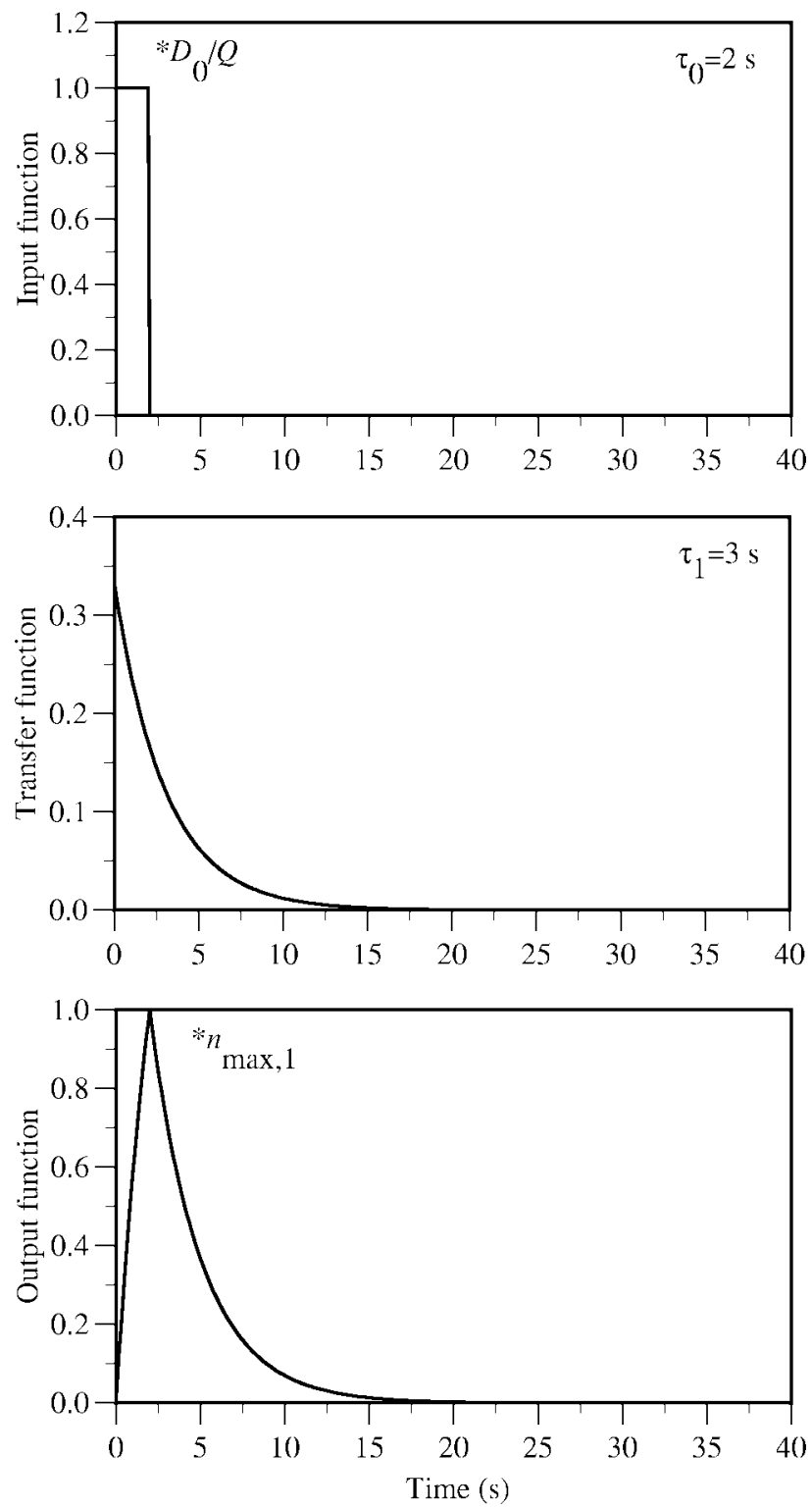

Fig. 3. The dilution curve of a simple mixing chamber after a rectangle-shaped injection. This input function is used to approximate the premixing of the contrast with a finite volume of fluid before reaching the mixing chamber. The output function is characterized by a period of wash-in period followed by a period of wash-out.

Using (2) again, the output function at the second mixing chamber is found to be as shown in (11) at the bottom of the previous page, where $\tau_{p}=\tau_{0}+\tau_{12}$, and $\tau_{12}=$ $\left[\left(\tau_{1} \tau_{2}\right) /\left(\tau_{2}-\tau_{1}\right)\right] \ln \left[\left(1-e^{-\tau_{0} / \tau_{1}}\right) /\left(1-e^{-\tau_{0} / \tau_{2}}\right)\right]$. The peak of $n_{2}(t)$ occurs at $t=\tau_{p}$, with a peak value e16

$$
\begin{aligned}
n_{\max , 2} & =\frac{D_{0}}{V_{1}+V_{2}} \frac{\tau_{1}+\tau_{2}}{\tau_{0}} \frac{\left[1-e^{-\tau_{0} / \tau_{1}}\right]^{-\tau_{1} /\left(\tau_{2}-\tau_{1}\right)}}{\left[1-e^{-\tau_{0} / \tau_{2}}\right]^{-\tau_{2} /\left(\tau_{2}-\tau_{1}\right)}} \\
& =\frac{D_{0}}{Q \tau_{0}} \frac{\left[1-e^{-\tau_{0} / \tau_{1}}\right]^{\tau_{1} /\left(\tau_{2}-\tau_{1}\right)}}{\left[1-e^{-\tau_{0} / \tau_{2}}\right]^{-\tau_{2} /\left(\tau_{2}-\tau_{1}\right)}}
\end{aligned}
$$

Once $n_{2}(t)$ is determined, the volume flow rate can be calculated from its time integral using (1). From the appearance of (11), it is evident that the time constants $\tau_{1}$ and $\tau_{2}$ cannot be calculated reliably from the concentration curve $n_{2}(t)$ alone.
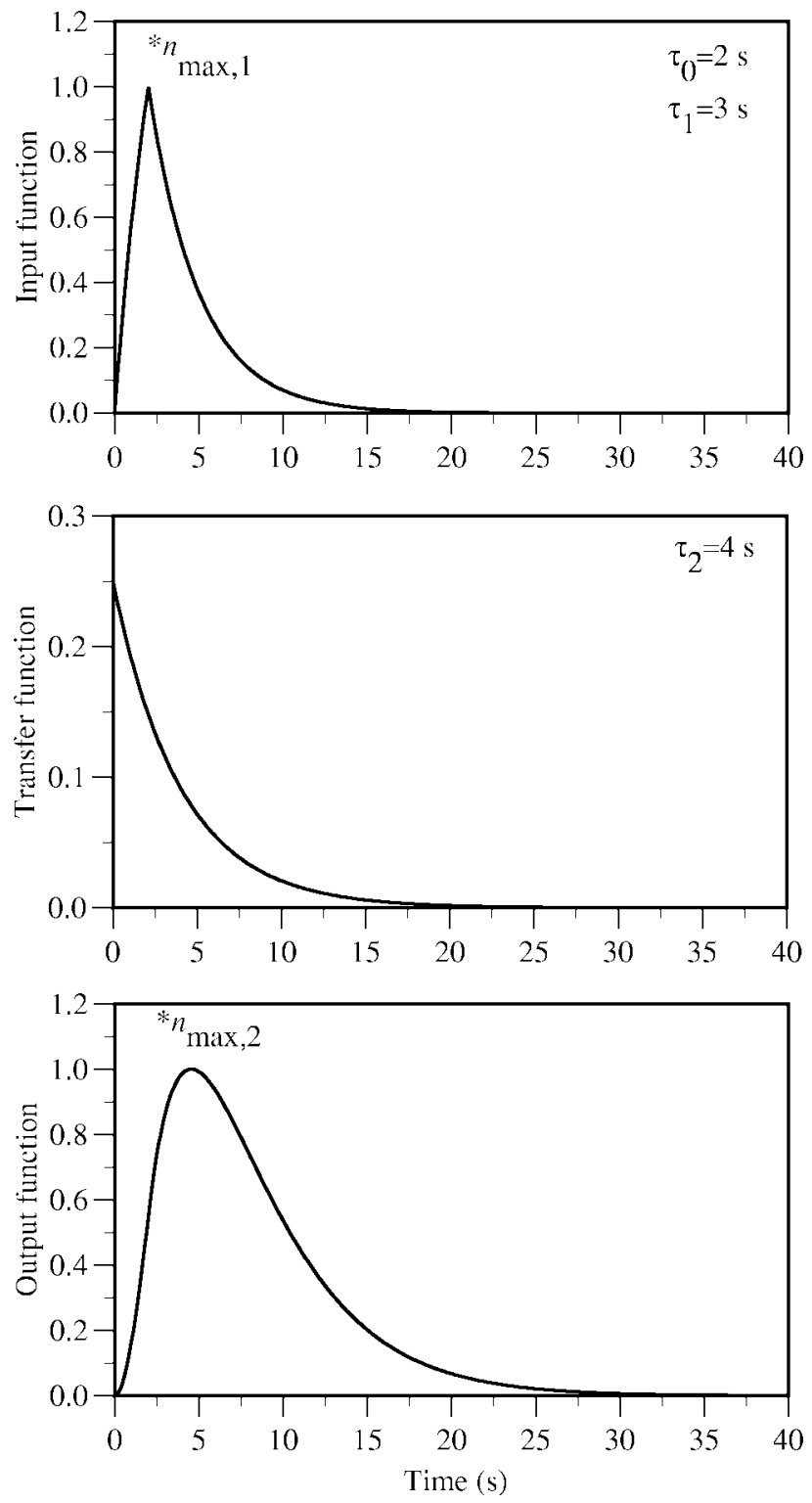

Fig. 4. The dilution curve of two simple mixing chambers after a rectangle-shaped injection.

However, if the concentration curves at the input and output are measured simultaneously, the centroid of each of the curves can be calculated. We would have the time constant $\tau_{2}=\bar{t}_{2}-\bar{t}_{1}$, from which the volume of distribution $V_{2}$ is calculated from $V_{2}=\tau_{2} Q$.

\section{Effect of Recirculation}

When recirculation is allowed, the dilution theory becomes much more complicated. Let us limit the discussion to the first mixing chamber. Before recirculation begins, the concentration of the agent is given by (9). After many recirculation paths, the concentration reaches its limiting value, which is given $n_{\infty}(t)=D_{0} / V_{T}$, where is $V_{T}$ the total volume the contrast has been mixed with (total blood volume). Fig. 5(a) shows the hypothetical curve as a function of time. This phase of the contrast dilution curve may or may not occur depending on the properties of the contrast agent, as discussed below. 


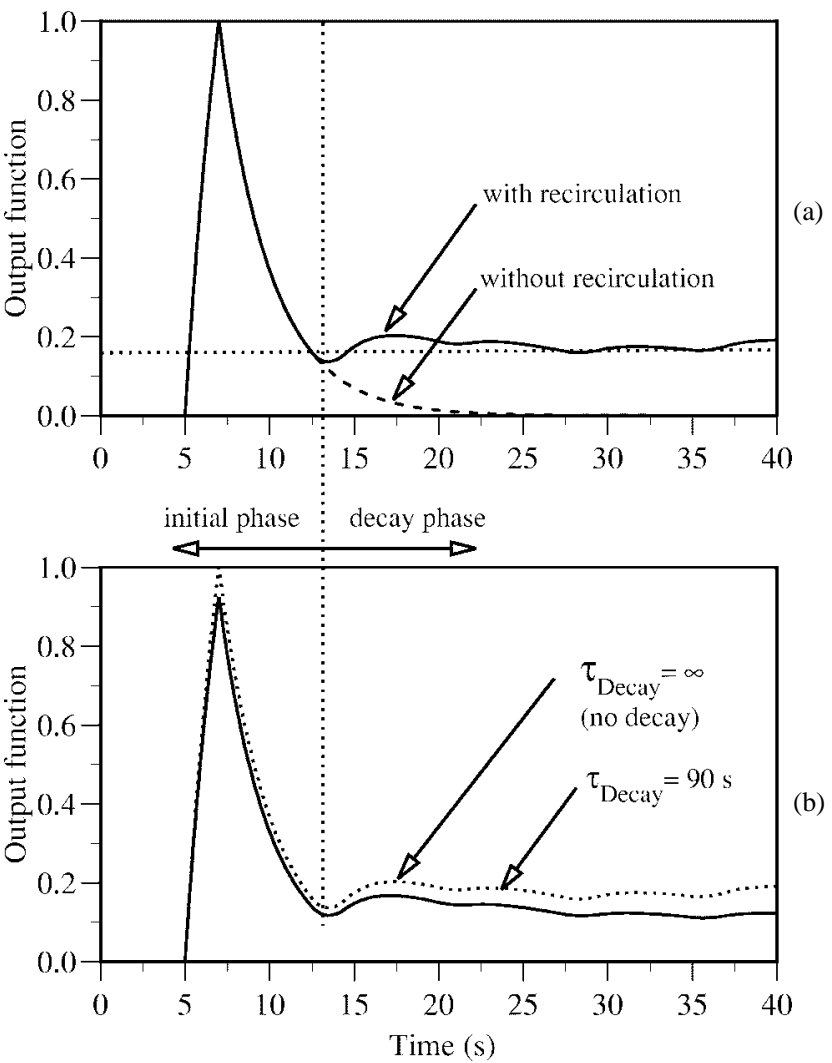

Fig. 5. The effect of recirculation of (a) the contrast agent and (b) the decay of the agent.

\section{Effect of Decay}

The condition that the total amount of the contrast remains in circulation is seldom, if ever, achieved. For ultrasound studies, the measured backscatter intensity depends on the acoustic scattering and attenuation properties of the contrast agent, among other parameters. The scattering and attenuation properties for bubble-based ultrasound contrast agent in turn depend on the size distribution and the number density of the bubbles. While various stabilizing agent or mechanism is incorporated in each type of ultrasound contrast agent, some bubbles might be destroyed by mechanical or chemical means after the agent is injected into the circulatory system of the patient. Some bubbles might be eliminated from circulation simply because their size is comparable to the size of capillary. Some bubbles might disintegrate under cardiac pressure. The effectiveness of ultrasound contrast agent is affected by these effects. To simplify discussions, we define a decay constant $\tau_{\text {decay }}$, which combines the effect of elimination of the agents from circulation by the body and the decay of the agent (natural and/or pressure dependent), as well as the possible change of size distribution. This is incorporated into the earlier discussions by modifying each of the effective concentration curves by a time-dependent multiplicative factor $e^{-t / \tau_{\text {Dccay }}}$. For example, the effective concentration of the agent during the initial phase of the dilution curve can now be written as $n_{1}^{\prime}(t)=n_{1}(t) e^{-t / \tau_{\text {Docay }}}$, where $n_{1}(t)$ is the concentration of the agent without the effect of decay. Notice that the maximum value of $n_{1}^{\prime}(t)$ is less than $n_{\max , 1}$. If $\tau_{\text {Decay }} \gg \tau_{1}$, the effect of the decay can be ignored when studying the first pass kinetics of the contrast agent dilution.

The last phase of dilution, shown in Fig. 5(b), is now $n_{\infty}^{\prime}(t)=\left(D_{0} / V_{T}\right) e^{-t / \tau_{\text {Dccay }}}$. Due to the decay phenomenon shown in Fig. 5(b), the dilution curve is sometimes divided into the initial phase and the decay phase.

\section{E. Numerical Considerations}

From the above discussion, it is clear that, for the calculation of flow rate, the initial portion of dilution curve may not be completely unaffected by recirculation effects, as is the case for in vivo studies. In this instance, models such as those given above are necessary to predict the complete history of the dilution curve from partial measurement of the curve (the initial phase) and from which to calculate the volume flow rate. Models given in (9) and (11) are relatively simple, so numerical fitting of the initial phase data can be achieved. Alternate models can be constructed, however, which are behaved better numerically. It must be noted that any such model must obey the mass conservation law given by (1). The gamma function, defined as

$$
n_{i}(t)= \begin{cases}0, & t<0 \\ n_{\max , i}\left(t / \tau_{p, i}\right)^{\tau_{p, i} / \tau_{w, i}} e^{-\left(t-\tau_{p, i}\right) / \tau_{w, i},} & t \geq 0 .\end{cases}
$$

has been chosen by many researchers for this purpose. The three parameters used are the peak concentration $n_{\max , i}$, time to peak concentration $\tau_{p, i}$, and wash-out constant $\tau_{w, i}$. The peak of $n_{i}(t)$ occurs at $t=\tau_{p, i}$, with a peak value given by

$$
n_{\max , i}=\frac{D_{0}}{Q} \frac{\left(\tau_{p, i} / \tau_{w, i}\right)^{\tau_{p, i} / \tau_{w, i}} e^{-\tau_{p, i} / \tau_{w, i}}}{\left(\tau_{p, i} / \tau_{w, i}\right) ! \tau_{w, i}} .
$$

Once the parameters $n_{\max , i}, \tau_{p, i}$, and $\tau_{w, i}$ are estimated, the volume flow rate can be calculated directly from this expression, i.e.,

$$
Q=\frac{D_{0}}{n_{\max , i}} \frac{\left(\tau_{p, i} / \tau_{w, i}\right)^{\tau_{p, i} / \tau_{w, i}} e^{-\tau_{p, i} / \tau_{w, i}}}{\left(\tau_{p, i} / \tau_{w, i}\right) ! \tau_{w, i}} .
$$

It can be shown that the centroid of the concentration curve given by the gamma function is $\bar{t}_{i}=\tau_{p, i}+\tau_{w, i}$. If the concentration curves at the input and output of a mixing chamber are measured simultaneously, then the centroid of each of the curves can be calculated. From (5), we would have the transit time of the mixing chamber, i.e., $\tau_{i}=\bar{t}_{i}-\bar{t}_{i-1}$, from which the volume of distribution is calculated from $V_{i}=\tau_{i} Q$.

\section{UltRASOUND MEASUREMENTS}

Quantitative ultrasound sound measurements can be used to measure the dilution curve of a contrast agent indirectly. A simplified schematic diagram of such a measurement system is shown in Fig. 6. The backscattered ultrasound intensity can be expressed as [4]

$$
P_{s}(t)=X_{s} A_{s}(t) \eta_{s}(t)
$$

where $P_{s}(t)$ is the detected backscatter ultrasound power from a selected region-of-interest (ROI) in tissue, $X_{s}$ is the 


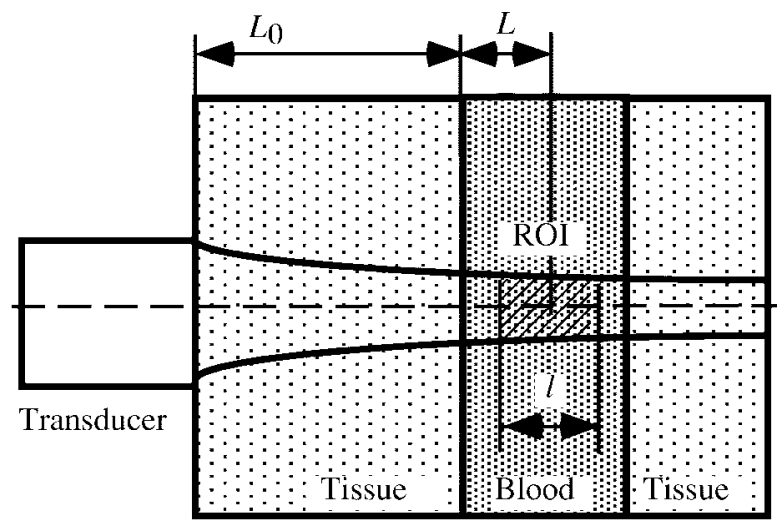

(a)

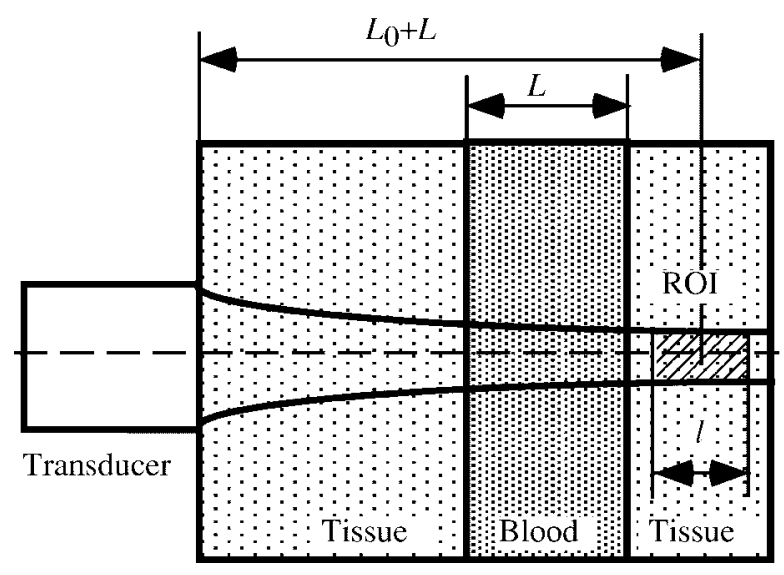

(b)

Fig. 6. A schematic diagram of a typical ultrasound measurement arrangement. The mean distance from the transducer to the sample volume is $L_{0}+L$. (a) The acoustic sample volume is in the blood pool and (b) The acoustic sample volume is in the tissue beyond the blood pool.

combined system transfer function, $A_{s}(t)$ accounts for the loss of transmitted and scattered ultrasound energy due to attenuation, and $\eta_{s}(t)$ is the backscatter coefficient of the tissue with or without the contrast agent. Here the combined system transfer function $X_{s}$ includes the effect of the diffraction pattern of the transducer, the effect of the pulser and receiver, as well as the effect of any gain function and the spherical spreading of the scattered acoustic energy.

The backscatter coefficient of a scattering medium is a statistical parameter which describes the complex process of scattering of ultrasound energy by the inhomogeneities within a medium (or tissue). A vast amount of literature is available on this topic [5]-[8]. If the concentration of contrast agents in the blood is relatively low during the measurements, it can be assumed that the backscatter coefficient is an additive parameter, i.e.,

$$
\eta_{s}(t)=\eta_{\mathrm{BL}}+\eta_{C}(t)
$$

where $\eta_{\mathrm{BL}}$ is the baseline backscatter coefficient of the blood or tissue without the contrast agent, and $\eta_{C}(t)$ is the backscatter coefficient of the contrast agent alone. The backscatter coefficient of the contrast agent has been proven to be proportional to the concentration of the agent in relatively low concentrations [9]-[11], i.e.,

$$
\eta_{C}(t)=\sigma_{d} \cdot n(t)
$$

where $n(t)$ is the number density of the contrast agent (number of bubbles per unit volume), and $\sigma_{d}$ is the differential backscatter cross section of a single bubble. We will use $n(t)$ to express generically the concentration of the contrast agent, be it the number density or mass concentration, and hence $\sigma_{d}$ is treated as a proportionality constant, the physical meaning of which is determined by the units of $n(t)$. When $n(t)$ is the mass concentration, $\sigma_{d}$ is the differential backscatter cross section per unit mass of the contrast agent.

The attenuation correction can be written as

$$
A_{s}(t)=e^{-4 \alpha_{0} L_{0}-4 \alpha(t) L}
$$

where $\alpha_{0}$ is the attenuation coefficient of the tissue layer, $L_{0}$ is the depth of tissue layer between the transducer and the blood vessel, $\alpha(t)$ is the attenuation coefficient of the blood-contrast mixture, and $L$ is the mean depth of the ROI measured from the tissue wall. The overall depth of the ROI measured from the transducer is therefore $L_{0}+L$.

Similar to the backscatter coefficient, it can be assumed that the attenuation coefficient of the blood-contrast mixture is an additive parameter, i.e.,

$$
\alpha(t)=\alpha_{\mathrm{BL}}+\alpha_{C}(t)
$$

where $\alpha_{\mathrm{BL}}$ is the attenuation coefficient of the blood without the addition of contrast agents, and $\alpha_{C}(t)$ is the additional attenuation due to the presence of contrast agents. It has been confirmed experimentally that the attenuation coefficient is proportional to the concentration of the contrast agent when the concentration is relatively low, [11] i.e.,

$$
\alpha_{C}(t)=\sigma_{a} \cdot n(t)
$$

where $\sigma_{a}$ is the attenuation cross section (commonly referred to as extinction cross section) of a single bubble. The attenuation cross section can be considered of as the sum of two terms, the absorption cross section and the total scattering cross section (ultrasound energy loss due to scattering) [12]. Since the contrast agent is a relatively strong scatterer by design, it is assumed that the total scattering cross section dominates the attenuation cross section (i.e., the absorption cross section is negligible compared with the total scattering cross section). The total scattering cross section of a single bubble is equal to the spatial integral of the differential scattering cross section. When the size of the bubbles in the contrast agent is smaller than the wave length, the total scattering cross section is $4 \pi \sigma_{d}$. This relationship can be proven numerically for imaging frequencies $(2.5-10 \mathrm{MHz})$ and clinically usable bubble sizes (less than $8 \mu \mathrm{m}$ in diameter). Under these assumptions, we have $\sigma_{a}=4 \pi \sigma_{d}$. For contrast agents like Albunex where a membrane is used to encapsulate a bubble, the absorption cross section might not be negligible compared with the total scattering cross section, the attenuation cross section is larger than $4 \pi \sigma_{d}$ in the frequency and size ranges mentioned earlier. 


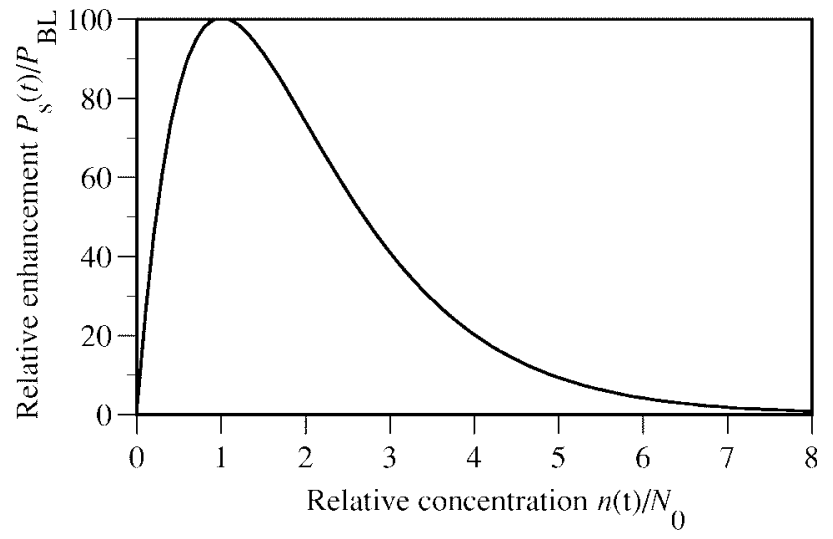

Fig. 7. Simulation of the effect of attenuation on the ultrasound measurement. $N_{0}$ is the optimal concentration (see text). $u=3.69 \times 10^{-3}$ is used in the simulation such that the maximum possible enhancement in backscatter intensity is 100 , or $20 \mathrm{~dB}$.

The exact relationship between the attenuation cross section and scattering cross section expressed above is not necessary for the following discussion. However, it serves as a guideline when the effect of attenuation is considered.

Combining (16)-(21), we have

$$
P_{s}(t) / P_{\mathrm{BL}}=e^{-4 L \sigma_{a} n(t)}\left[1+\left(\sigma_{d} / \eta_{\mathrm{BL}}\right) n(t)\right]
$$

where $P_{\mathrm{BL}}=X_{s} \eta_{\mathrm{BL}} e^{-4 \alpha_{0} L_{0}-4 \alpha_{\mathrm{BL}} L}$ is the baseline backscattered ultrasound power from the blood pool when no contrast agent is present, i.e., $n(t)=0$. It is realized that if the relative enhancement of the backscatter intensity can be monitored quantitatively as a function of time, the concentration of the contrast agent, and hence the mixing volume and the volume flow rate, can be calculated.

Notice that the effect of attenuation limits the maximum possible enhancement that any ultrasound contrast agent can provide. The maximum possible enhancement can be found to be $P_{s 0} / P_{\mathrm{BL}}=e^{-1+u} / u$ when the optimal concentration is reached, where $u$ is a nondimensional parameter defined as $u=4 L \eta_{\mathrm{BL}}\left(\sigma_{a} / \sigma_{d}\right)$. The optimal concentration is given by $N_{0}=((1 / u)-1)\left(\eta_{\mathrm{BL}} / \sigma_{d}\right)$. For contrast enhancement it is desirable to have a small value of $u$. It is interesting to note that $u$ mainly depends on the background scattering properties and the depth at which the measurement is made. For example, for blood at $5 \mathrm{MHz}$ and 1-cm sample depth, we find $u=3.47 \times 10^{-3}$, so the maximum possible enhancement any contrast agent can provide is $P_{s 0} / P_{\mathrm{BL}}=128$, or $21 \mathrm{~dB}$. On the other hand, for liver at $5 \mathrm{MHz}$ and 1-cm sample depth, we find $u=0.151$, so the maximum possible enhancement any homogeneously distributed contrast agent can provide is $P_{s 0} / P_{\mathrm{BL}}=2.8$, or $4.5 \mathrm{~dB}$. This indicates that ultrasound contrast agents for liver imaging might not work properly, regardless of the design of the agent, unless the agent targets certain types of cells such that an inhomogeneous distribution of the agent is realized. Such an agent has been reported [13].

The effect of attenuation on the measured backscatter intensity is shown in Fig. 7, where $u=3.69 \times 10^{-3}$ is used in the simulation such that the maximum possible enhancement in backscatter intensity is 100 , or $20 \mathrm{~dB}$.
The same effect also governs the shape of intensity-time curves. This is demonstrated by the simulation in Fig. 8 . For relatively small dose injections such that $n_{\max }<N_{0}$, the relative enhancement suffers a loss, but the overall shape of the intensity-time curve remains unchanged. The maximum enhancement is equal to $P_{s, \max } / P_{\mathrm{BL}}=(1+$ $\left.n_{\max } \sigma_{d} / \eta_{\mathrm{BL}}\right) e^{-4 L \sigma_{a} \cdot n_{\max }}$, which occurs when the peak concentration is reached [Fig. 8(a)]. For higher injected doses such that $n_{\max }>N_{0}$, the relative enhancement suffers a loss, and the overall shape of the intensity-time curve also changes. There is a local minimum at peak concentration, with an amplitude $P_{s, \min } / P_{\mathrm{BL}}=\left(1+n_{\max } \sigma_{d} / \eta_{\mathrm{BL}}\right) e^{-4 L n_{\max } \sigma_{a}}$. Two local maxima of the same magnitude occur, one on each side of the peak concentration, where the magnitude is $P_{s, \text { max }} / P_{\mathrm{BL}}=e^{-1+u} / u$ [Fig. 8(b)]. This magnitude is not determined by the actual dose of injection, but by the relative scattering and attenuation properties of the contrast agent, as indicated earlier. For an even higher injected dose such that $n_{\max }>\left[\ln \left(1+n_{\max } \sigma_{d} / \eta_{\mathrm{BL}}\right) / 4 L \sigma_{a}\right]$, negative enhancement occurs in the vicinity of peak concentration, i.e., $P_{s, \min } / P_{\mathrm{BL}}<1$ [Fig 8(c)].

From (22), the concentration of the contrast agent can be calculated from the relative measurement of backscatter intensity, i.e.,

$$
n(t)=\left(\eta_{\mathrm{BL}} / \sigma_{d}\right)\left\{\left[P_{s}(t) / P_{\mathrm{BL}}\right] e^{4 L \sigma_{a} n(t)}-1\right\}
$$

An iterative method can be used to solve the above equation. Both the scattering and the attenuation cross sections of the contrast agent are required to obtain the absolute measure of the concentration curve. If the effect of attenuation is known to be small for the whole duration of the dilution study, however, (23) becomes

$$
n(t) \approx\left(\eta_{\mathrm{BL}} / \sigma_{d}\right)\left[P_{s}(t) / P_{\mathrm{BL}}-1\right] .
$$

In this case, only the scattering cross section of the contrast agent is required. When calculating the centroid of the concentration curve using (4), neither the scattering cross section nor the attenuation cross section are required, as long as the linearity prescribed by (18) and (21) are satisfied.

Alternatively, the backscatter power at a depth just beyond the contrast enhanced zone (like the blood vessel) can be used, as shown in Fig. 6(b). The backscatter coefficient here is not affected by the presence of the contrast agent in the blood pool, i.e., $\eta_{s}(t)=\eta_{\mathrm{BL}}$. The Attenuation correction function, however, can still be written as (21). Using (16) again, we have

$$
P_{s}(t) / P_{\mathrm{BL}}=e^{-4 L \sigma_{a} n(t)}
$$

where $P_{\mathrm{BL}}=X_{s} \eta_{\mathrm{BL}} e^{-4 \alpha_{0} L_{0}-4 \alpha_{\mathrm{BL}} L}$ is the baseline backscattered power from the tissue when the contrast agent is absent. Therefore, it is possible to measure the contrast dilution curve by measuring the decrease of the backscatter signal due to the contrast injection, i.e.,

$$
n(t)=-\frac{1}{4 L \sigma_{a}} \ln \left[P_{s}(t) / P_{\mathrm{BL}}\right]
$$

Only the attenuation cross section of the contrast agent is required. 

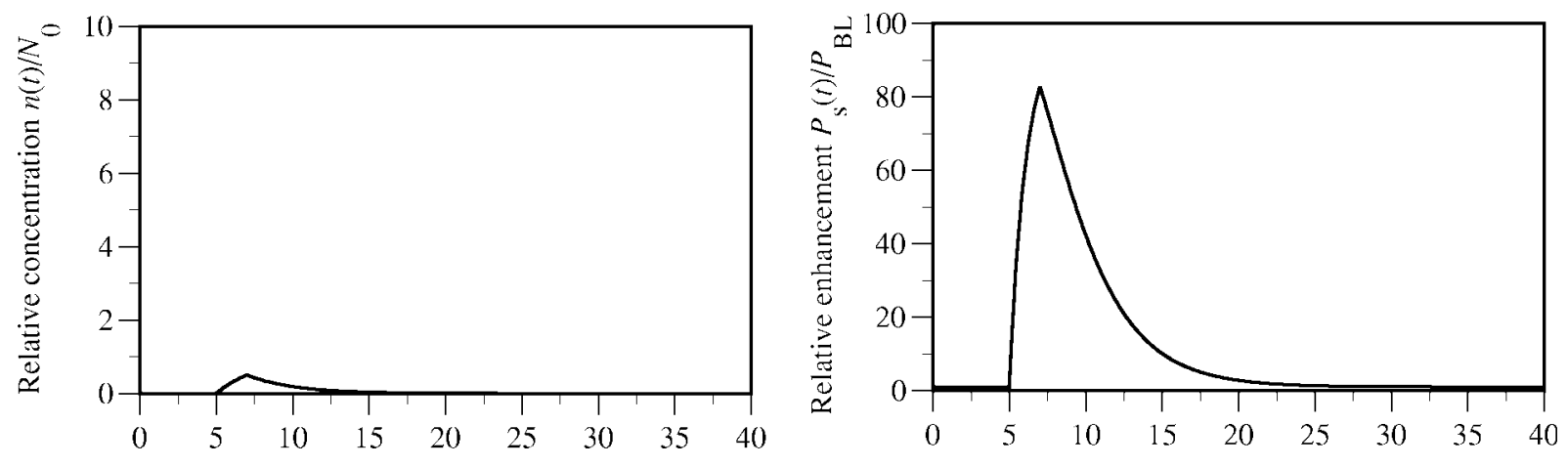

(a)
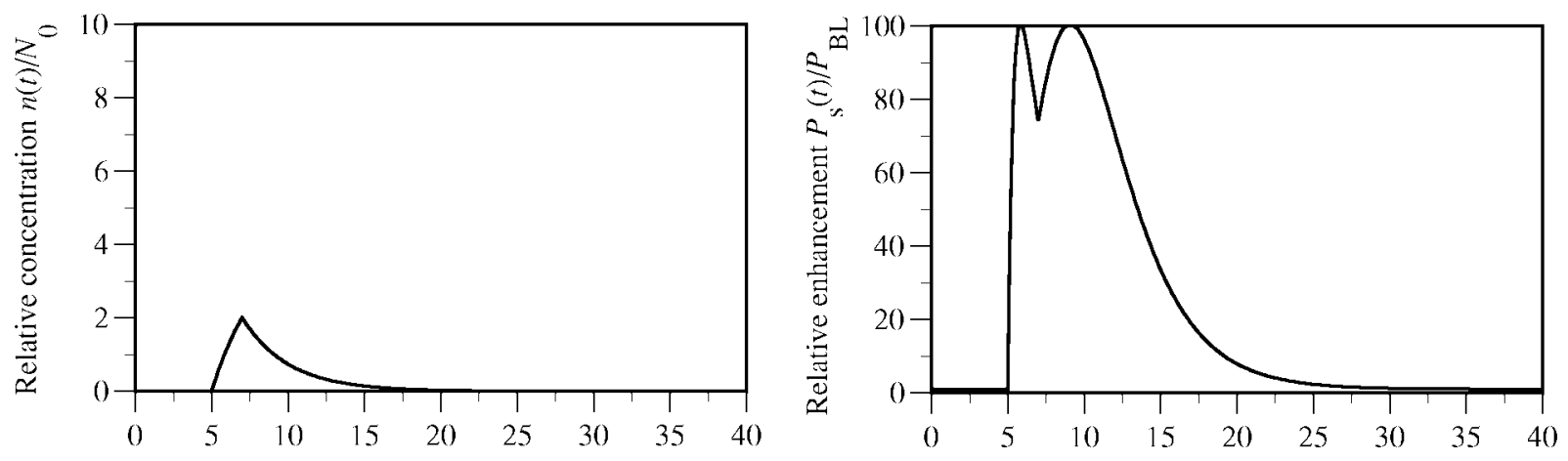

(b)
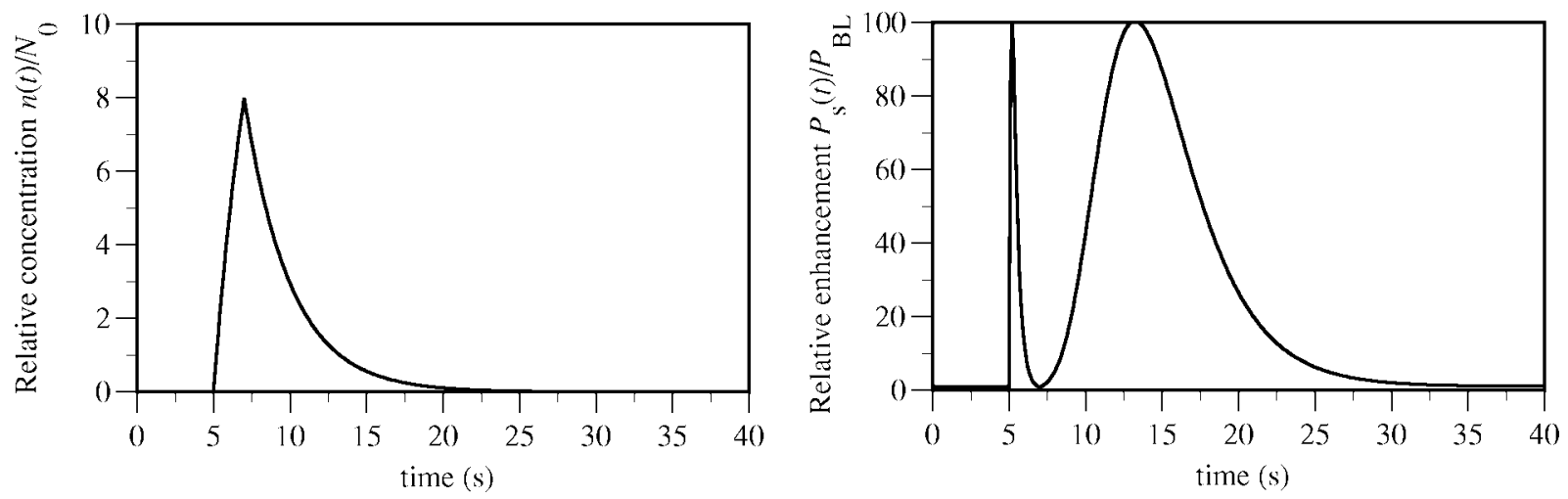

(c)

Fig. 8. Simulation of the effect of attenuation on the measured intensity-time curve. The relative concentration curves are shown on the left, and the relative enhancement of the backscattered intensity as functions of time on the right. (a) $n_{\max }=N_{0} / 2$, (b) $n_{\max }=2 N_{0}$, and (c) $n_{\max }=8 N_{0}$. $N_{0}$ is the optimal concentration (see text), $n_{\max }$ is the peak concentration. $u=3.69 \times 10^{-3}$ is used in the simulation such that the maximum possible enhancement in backscatter intensity is 100 , or $20 \mathrm{~dB}$.

The above discussion assumes that the backscatter and the attenuation coefficients are directly proportional to the concentration of the contrast agent. While this has been proven by many experimental studies, the assumptions are valid only in a limited range of concentrations. When the concentration of the contrast increases, multiple scattering plays a more important role and the relationship between the backscatter coefficient and concentration of the contrast becomes nonlinear. This phenomenon will be demonstrated by the experimental results that follow.

\section{EXPERIMENTAL RESULTS}

An experiment was performed to test the theory presented above. A schematic diagram of the experimental apparatus is shown in Fig. 9. A gated tone burst, generated by a function generator (Model HP 8116A, Hewlet-Packard GmbH, Germany) and amplified $55 \mathrm{~dB}$ (Model A150, ENI Inc., Rochester, NY), was used to excite the focused transducer. The transducer used was a focused cylindrical transducer (Krautkramer Branson, Lewistown, PA), with an active element $a=6.35 \mathrm{~mm}$ in radius, and a geometrical focus $r_{0}=$ $50.8 \mathrm{~mm}$. Via a transmit/receive (T/R) switch (Model RDX6, RITEK Inc., Warwick, RI), the backscattered ultrasound signal was preamplified and connected to a digitizing oscilloscope (Model 9430, LeCroy Corporation, Chestnut Ridge, NJ). The radio-frequency (RF) signal was digitized at a $50-\mathrm{MHz}$ sampling rate with 10-b precision. An antialiasing filter with a cutoff frequency of $45 \mathrm{MHz}$ was used before digitization. The digitized RF signal was transferred via an IEEE 488 


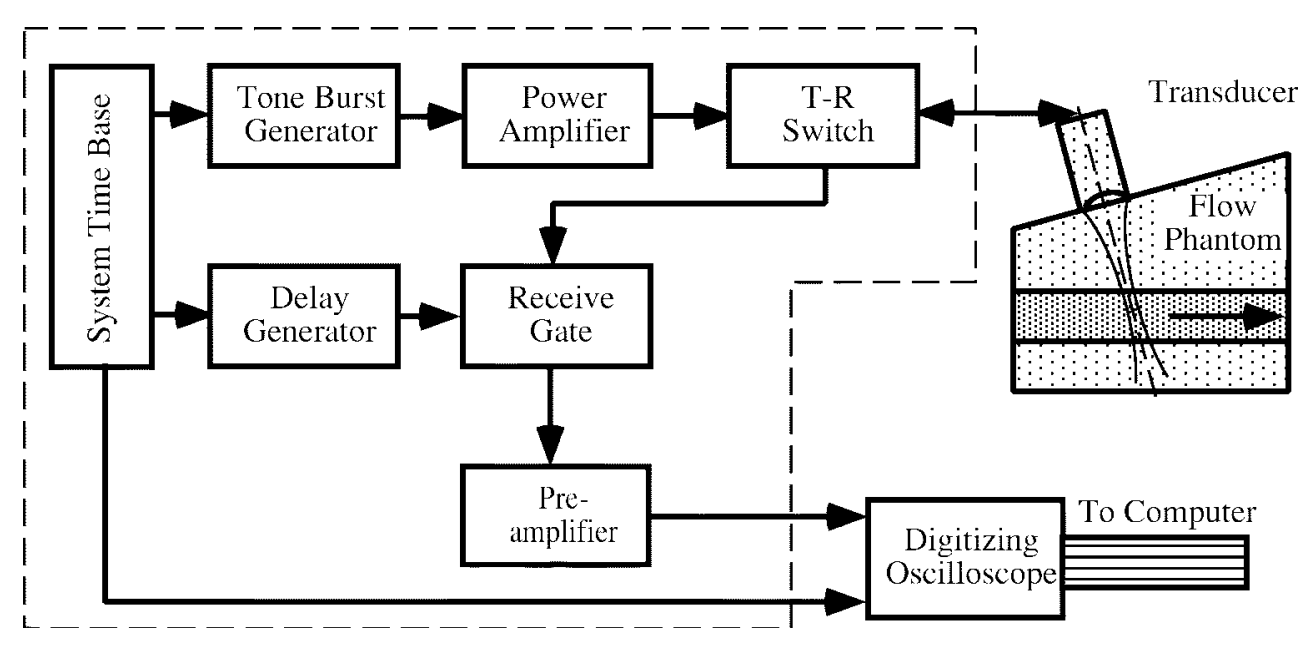

Fig. 9. Apparatus for the backscatter measurement system.

TABLE I

SuMmary OF EXPERIMENTAL RESUlTS

\begin{tabular}{lrrr}
\hline & Group A & Group B & Group C \\
\hline Dose $D_{0}(\mathrm{mg})$ & 150 & 150 & 300 \\
Flow rate $Q\left(\mathrm{~cm}^{2}\right.$ min_1 & $308 \pm 3$ & $610 \pm 2$ & $309 \pm 3$ \\
$D_{0} / Q\left(\mathrm{mg} \mathrm{cm}^{-3} \mathrm{~s}\right)$ & $29.3 \pm 0.2$ & $14.7 \pm 0.1$ & $58.3 \pm 0.5$ \\
$\tau_{p}(\mathrm{~s})$ & $12.1 \pm 1.2$ & $4.05 \pm 0.16$ & $11.0 \pm 0.9$ \\
$\tau_{w}(\mathrm{~s})$ & $14.7 \pm 0.4$ & $6.45 \pm 0.08$ & $19.6 \pm 0.5$ \\
Transit time $\tau(\mathrm{s})$ & $20.7 \pm 0.5$ & $8.48 \pm 0.10$ & $25.1 \pm 0.4$ \\
Peak Concentration $n_{\max }\left(\mathrm{mg} \mathrm{cm}^{-3}\right)$ & $0.80 \pm 0.05$ & $1.16 \pm 0.10$ & $1.23 \pm 0.4$ \\
Integral under concentration curve $I\left(\mathrm{mg} \mathrm{cm}^{-3 s}\right)$ & $29.3 \pm 1.9$ & $16.8 \pm 1.0$ & $52.0 \pm 2.3$ \\
Volume of distribution $V\left(\mathrm{~cm}^{3}\right)$ & $106 \pm 3$ & $86.2 \pm 1.1$ & $129 \pm 2$ \\
\hline
\end{tabular}

interface to a personal computer for storage and processing. The envelope of the RF signal was calculated from the amplitude of the complex analytic function associated with the RF signal.

The flow phantom is a tissue equivalent phantom (TEP) (ATS, Bridgeport, CT), which has background scattering and attenuation properties similar to liver, as specified by the American Institute of Ultrasound in Medicine (AIUM). A built-in flow channel with an diameter of $8 \mathrm{~mm}$ was used for imaging. The flow was driven by gravity. The contrast agent was premixed as described below. Injectant passed through a mixing chamber before reaching the flow channel. The hydrodynamic pressure inside the flow channel was monitored with a pressure transducer (Model 1290A, Hewlett-Packard, Palo Alto, CA) and monitor (Model 56, Hewlett-Packard, Palo Alto, CA). The nominal pressure in the flow channel was 55 $\mathrm{mmHg}$. The volume flow rate was monitored with a flow meter (Model T206, Transonic Systems, Inc., Ithaca, NY).

Three groups of injections were made. The dosages of the contrast agent were $150 \mathrm{mg}$ and $300 \mathrm{mg}$, and the volume flow rates used were $300 \mathrm{~cm}^{3} / \mathrm{min}$ and $600 \mathrm{~cm}^{3} / \mathrm{min}$, as listed in Table I. The ultrasound contrast used was SH U 508 A (Schering AG, Germany). The contrast agent was premixed at $300 \mathrm{mg} \mathrm{cm}^{-3}$ and allowed to stand for $2 \mathrm{~min}$ before injection. For Group A and Group B, the contrast agent was further diluted to $150 \mathrm{mg} \mathrm{cm}{ }^{-3}$ and a volume of $1 \mathrm{~cm}^{3}$ was injected. For Group C, a volume of $1 \mathrm{~cm}^{3}$ at $300 \mathrm{mg} \mathrm{cm}^{-3}$ was injected.
Shown in Fig. 10 are a single trace of RF signal from the ultrasound system [Fig. 10(b)] as well as the calculated $M$ mode image from a sequence of RF signals [Fig. 10(a)]. Three ROI's were chosen for analysis. ROI 1 is the proximal portion of the flow channel, ROI 2 is the distal portion of the flow channel, and ROI 3 is inside the TEP just beyond the flow channel. Relative intensity-time curves from these ROI's for one injection are shown in Fig. 11. As is apparent from these curves, the attenuation effect discussed earlier cannot be ignored when calculating the area under the intensity-time curve.

Fig. 12 shows the fitting of the intensity-time curve from ROI 3 to the gamma curve given in (14). The time constants obtained for this particular instance were $\tau_{p}=11.5 \mathrm{~s}$ and $\tau_{w}=15.0 \mathrm{~s}$. These were estimated without knowledge of the attenuation cross section of the contrast agent.

Since the dose of the contrast agent and the flow rate were both measured independently, it was possible to obtain the attenuation cross section of the contrast agent by using the mass conservation law, given by (1). Using this technique on Group A, we found $\sigma_{a}=0.25 \mathrm{~cm}^{2} \mathrm{mg}^{-1}$. Using this attenuation properties of the contrast agent, it was possible calculate the contrast dilution curves and the corresponding integrals under these curves from the relative backscatter intensity measurements from ROI 3. Results are summarized in Table I. The integrals under the concentration curves are also plotted against $D_{0} / Q$ in Fig. 13. Reasonable agreement with the mass conservation law is demonstrated. 


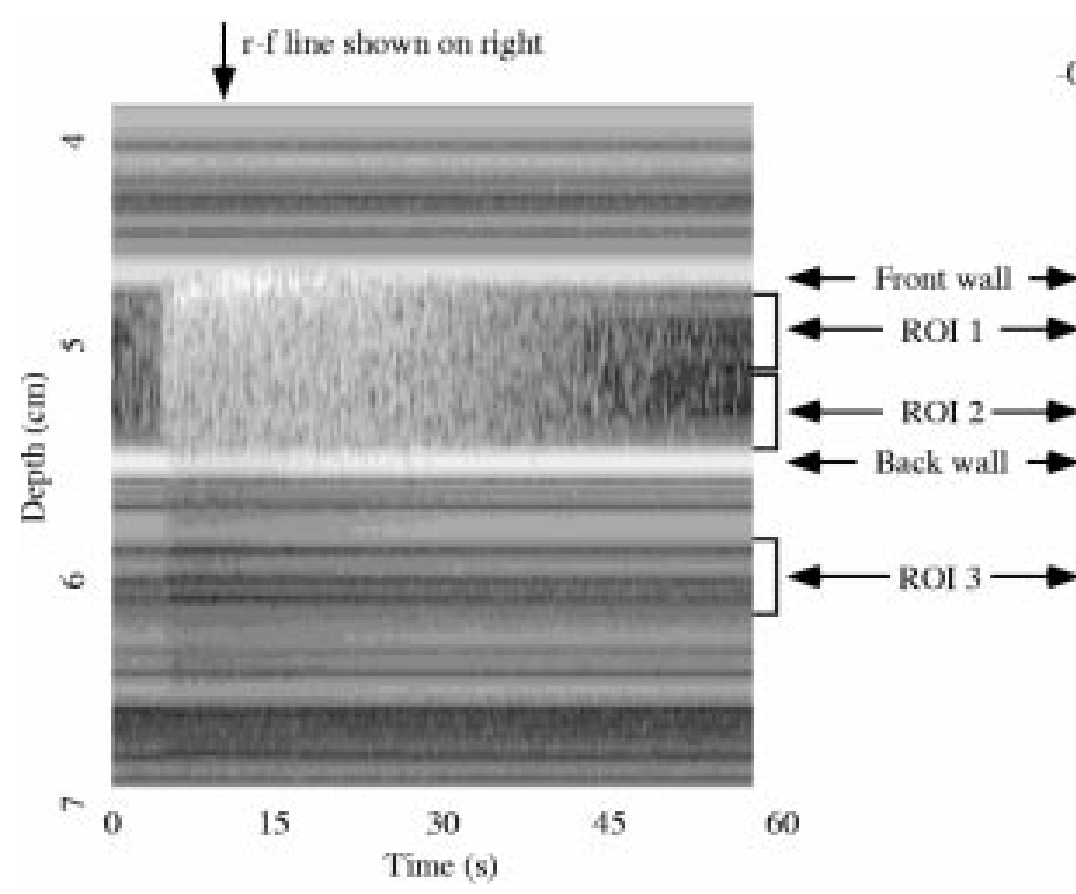

(a) (b)

Fig. 10. (b) Example radio frequency signal from the backscattering measurement and the definition of ROI. ROI 1 is from the proximal portion of the flow channel, ROI 2 is from the distal portion of the flow channel, and ROI 3 is from the TEP beyond the flow channel. (a) Calculated $M$-mode image. A $60-\mathrm{dB}$ dynamic range was used to display the $M$-mode image.

The centroid of the concentration curve was found from $\bar{t}=\tau_{p}+\tau_{w}$ when the gamma curve was used. If (9) was used instead, we would have $\bar{t}=\tau_{0} / 2+\tau$ where $\tau$ is the transit time of the mixing chamber. From Fig. (3), $\tau_{0}=\tau_{p}$. Therefore, the transit time can be calculated from $\tau=\tau_{p} / 2+\tau_{w}$.

The mean transit time for Group A was found to be $20.7 \mathrm{~s}$, which corresponds to a volume of distribution of $106 \mathrm{~cm}^{3}$. This volume is larger than the geometrical volume of the mixing chamber, which is about $90 \mathrm{~cm}^{3}$. Further mixing of the contrast agent between the mixing chamber and the measurement site occurred. For Group B with a higher flow rate, however, the mean transit time was found to be $8.48 \mathrm{~s}$, which corresponds to a volume of distribution of $86.2 \mathrm{~cm}^{3}$.

A close comparison between Group A and Group C revealed the nonlinear relationship between the attenuation coefficient and the concentration of the contrast agent at relatively high concentrations. Since the only difference between Group A and Group $\mathrm{C}$ was the dose of contrast agents used, the peak concentration was expected to be twice that of Group A, or $1.60 \mathrm{mg}$ $\mathrm{cm}^{-3}$. The measured peak concentration was an $23 \%$ under estimate. This can be explained by a nonlinear relationship between the attenuation coefficient and the concentration of the agent. As a result of this nonlinearity, the transit time was overestimated. The combined effect was a reasonable estimate of the integral under the concentration curve.

A high degree of repeatability within each group was observed. For example, the integral under the concentration curve had a standard variation to mean ratio of less than $6 \%$, while the transit time had a standard variation to mean ratio of less than $3 \%$.

Also shown in Fig. 11 were the expected relative backscatter using the calculated concentration curve in Fig. 12. Reason- able agreement was observed for ROI 1. For ROI 2 large deviation was observed, especially when the concentration was high. This indicates the effect of multiple scattering, and hence the nonlinear relationship between the backscatter coefficient and the concentration of the contrast agent, even at this modest level. It is estimated from Figs. 11 and 12 that a peak concentration of less than $0.3 \mathrm{mg} \mathrm{cm}^{-3}$ would insure the linearity between the backscatter coefficient and the concentration for this particular agent.

\section{Discussions AND CONCLUSION}

A mathematical model for the assessment of hemodynamic parameters using quantitative echocardiography has been presented where a simple models were used to represent the dilution of contrast agents in each mixing chamber. The experimental method involves the intravenous injection of an ultrasonic echo contrast agent. The relative enhancement of the backscattered ultrasound energy is measured as a function of time (the time-intensity curve). From this measurement, the concentration of the contrast agent is obtained. The mixing volume (the volume of distribution) and the volume flow rate (cardiac output) are calculated from the contrast dilution curve.

The backscatter coefficient of the contrast agent is linearly proportional to the concentration over a limited range. Above this range, the relationship between the backscatter coefficient of the contrast agent and its concentration become nonlinear. This makes it more difficult to calculate the dilution curve from the measured ultrasound backscatter signal. The attenuation coefficient of the contrast agent has a similar dependence on the concentration of the agent. 

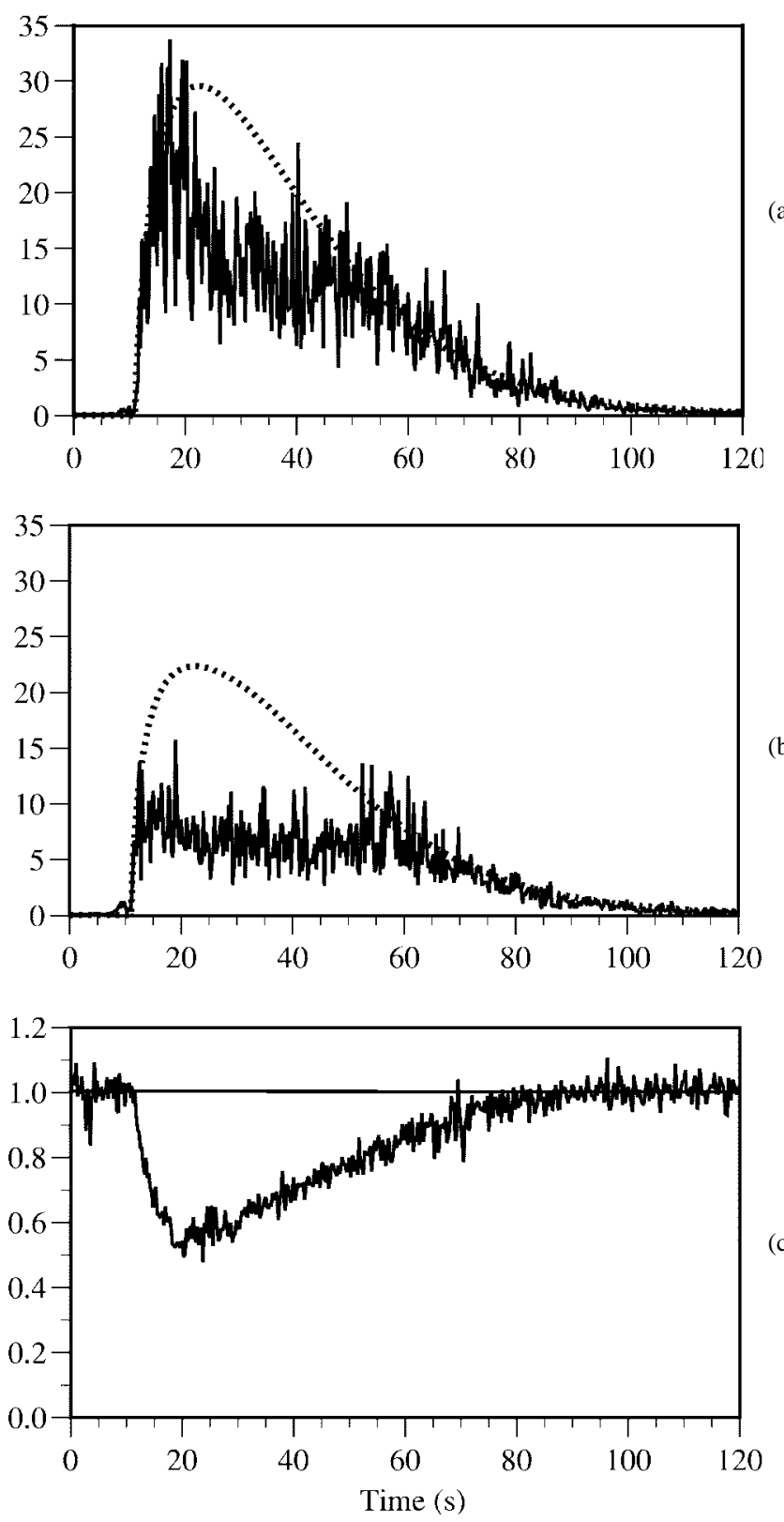

Fig. 11. Relative backscatter intensity-time curves calculated from the RF signal. (a) From ROI 1; (b) from ROI 2; (c) from ROI 3. Dashed lines in (a) and (b) represent the expected backscatter intensity due to the concentration curve shown in Fig. 12.

The effect of added attenuation due to the presence of the contrast agent plays an important role in the quantitative measurement of dilution curves. As indicated by (22), this effect may not be ignored unless the peak concentration of the agent is low enough.

The effect of added attenuation due to the presence of the contrast agent also limits the maximum ultrasound enhancement achievable. The maximum enhancement was found to dependent on the background scattering property of the medium into which the contrast agent will be mixed. This is usually blood, but may be soft tissue such as in the case of myocardial perfusion studies and liver imaging. It also depends on the length the ultrasound wave travels in the contrast enhanced medium.

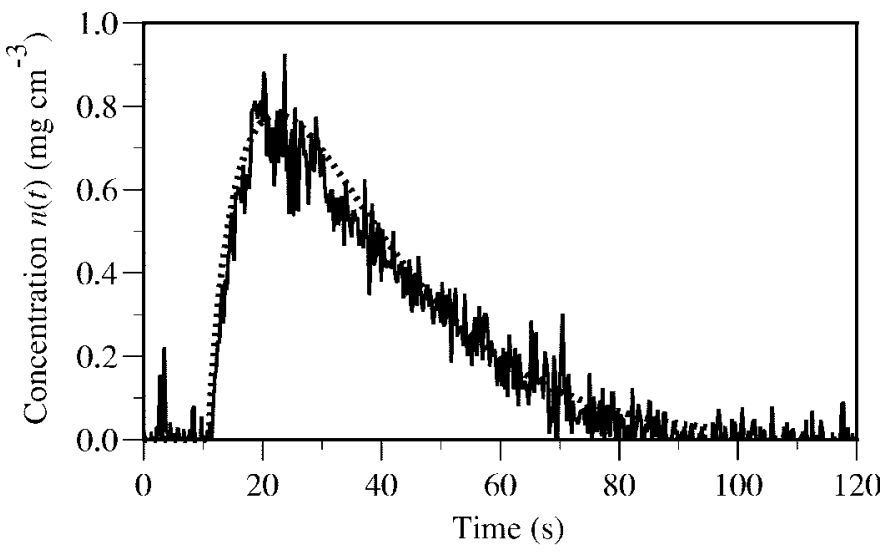

Fig. 12. The contrast concentration curve calculated from ROI 3. Solid line is calculated from the intensity-time curve shown in Fig. 11(c) by using (26), with $\sigma_{a}=0.25 \mathrm{~cm}^{2} \mathrm{mg}^{-1}$ and $L=0.8 \mathrm{~cm}$. Dashed line is fitted gamma distribution given in (13), with $n_{\max }=0.78 \mathrm{mg} \mathrm{cm}^{-3}, \tau_{p}=11.5 \mathrm{~s}$ and $\tau_{w}=15.0 \mathrm{~s}$.

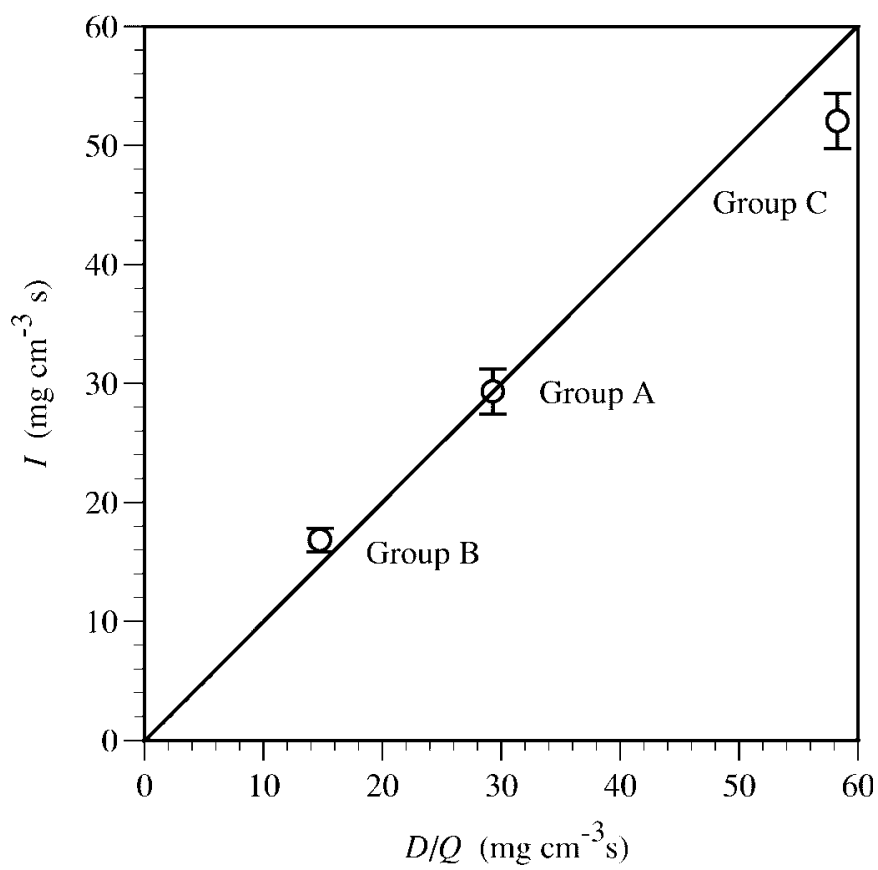

Fig. 13. The integral under the contrast concentration curves calculated from ROI 3 versus $D_{0} / Q$. Shown are group means \pm standard deviation. Measurements from Group A were used to obtained the attenuation cross section of the agent needed for the calculations, so the mean value of Group $\mathrm{A}$ is on the identity line.

Under limited conditions, it was possible to estimate the concentration of the contrast agent from the measured ultrasound backscatter intensity. Although nonintuitive, the best ROI for such an ultrasound measurement might be the soft tissue distal to, rather than in, the contrast enhanced blood pool. Choosing such an ROI, the measured change in the ultrasound backscatter intensity is related to the added attenuation coefficient of the contrast agent, rather than its added backscatter coefficient. This is similar to the greendye technique used clinically for the determination of cardiac output, where the added light absorption of the blood due to the green dye is used to measure the concentration of the indicator. 
In the experimental study presented, the RF signal was used to calculate the ultrasound intensity. Other means of detection are possible. An example is to use the video density measurement. Care must be taken to remove the effect of logarithmic compression commonly used for video display. Quantitative audio Doppler signal has also been reported to represent the backscatter intensity [14].

\section{REFERENCES}

[1] K. C. Ehlers, K. C. Mylrea, C. K. Waterson, and J. M. Calkins, "Cardiac output measurements. A review of current techniques and research," Ann. Biomed. Eng., vol. 14, pp. 219-239, 1986.

[2] S. B. Feinstein, P. Voci, L. J. Segil, and P. V. Harper, "Contrast echocardiography," in Cardiac Imaging, M. L. Marcus, H. R. Schelbert, D. J. Skorton, and G. L. Wolf, Eds. Philadelphia, PA: Harcourt Brace Jovanovich, 1991.

[3] K. Schwarz, X. Chen, G. Bezante, D. Phillips, and R. Schlief, "The Doppler kinetics of microbubble echo contrast agent," Ultrasound Med., Biol., vol. 22, pp. 453-462, 1996.

[4] X. Chen, D. Phillips, K. Q. Schwarz, J. G. Mottley, and K. J. Parker, "The measurement of backscatter coefficient from pulse-echo systems: A new formulation," IEEE Trans. Ultrason., Ferroelect., Freq. Contr., vol. 44, pp. 515-525, 1997.

[5] R. A. Sigelmann and J. M. Reid, "Analysis and measurement of ultrasonic backscattering from an ensemble of scatterers excited by sine-wave bursts," J. Acoust. Soc. Amer., vol. 53, pp. 1351-1355, 1973.

[6] J. A. Campbell and R. C. Waag, "Normalization of ultrasonic scattering measurements to obtain average differential scattering cross sections for tissues," J. Acoust. Soc. Amer., vol. 74, pp. 393-399, 1983.

[7] R. C. Waag, "A review of tissue characterization from ultrasonic scattering," IEEE Trans. Biomed. Eng., vol. BME-31, pp. 884-893, 1984.

[8] E. L. Madsen, M. F. Insana, and J. A. Zagzebski, "Method of data reduction for accurate determination of acoustic backscatter coefficients," J. Acoust. Soc. Amer., vol. 76, pp. 913-923, 1984.

[9] K. Schwarz, G. Bezante, X. Chen, R. Schlief, and K. Schiller, "Quantitative echo contrast measurement by Doppler sonography," Ultrasound Med., Biol., vol. 19, pp. 289-297, 1993.

[10] B. Wilson, K. K. Shung, B. Hete, H. Levene, and J. Barnhart, L., “A feasibility study on quantitating myocardial perfusion with Albunex ${ }^{\circledR}$, an ultrasonic contrast agent," Ultrasound Med., Biol., vol. 19, pp. 181-191, 1993.

[11] H. J. Bleeker, K. K. Shung, and J. L. Barnhart, "On the application of ultrasonic contrast agents for blood flowmetry and assessment of cardiac perfusion," J. Ultrasound Med., vol. 9, pp. 461-471, 1990.

[12] P. M. Morse and K. U. Ingard, Theoretical Acoustics. Princeton, NJ: Princeton Univ., 1986, ch. 8

[13] T. A. Tuthill, R. B. Baggs, M. R. Violante, and K. J. Parker, "Ultrasound properties of liver with and without particulate contrast agents," Ultrasound Med. Biol., vol. 8, pp. 490-498, 1991.

[14] K. Schwarz, G. Bezante, and X. Chen, "When can Doppler be used in place of integrated backscatter as a measure of scattered ultrasound intensity?," Ultrasound Med., Biol., pp. 231-242, 1995.

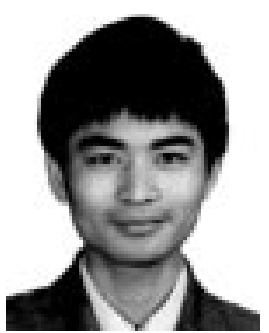

Xucai Chen was born in Jiangsu Province, China, on March 5, 1962. He finished his undergraduate training in China Mining Institute in 1982. He received the M.S., M.Phil., and Ph.D. degrees from Yale University, New Haven, CT, in 1986, 1989 , and 1991, respectively, in the Program in Engineering and Applied Science.

He is currently an Assistant Professor of Medicine and a Scientist in Electrical Engineering at the University of Rochester, Rochester, NY. He has been involved in the research of therapeutic and diagnostic ultrasound. He is interested in acoustic scattering, acoustic radiation force, and new ultrasound imaging modality for blood-flow visualization using ultrasound contrast agent.

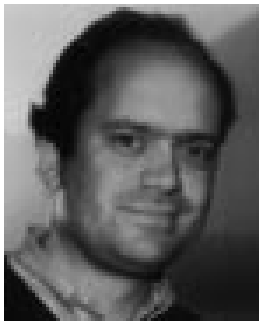

Karl Q. Schwarz was born on June 29, 1957. He received the A.B. degree in physics from Bowdoin College, Brunswick, ME, in 1979 and the M.D. degree from the University of Rochester, Rochester, NY, in 1983. He completed the internal medicine residency at Strong Memorial Hospital in Rochester, NY, in 1986 and the cardiology fellowship at the University of Rochester in 1989.

Since that time he has been a staff cardiologist at the University of Rochester and has been Director of the Echocardiography Laboratory since 1992 . He is currently an Associate Professor of Medicine and a Member of the Center for Biomedical Ultrasound in Rochester, NY. His primary research interest is in the behavior of small gas bubbles in a sound field. Recently, his research work has included harmonic imaging techniques and echo contrast. As Director of the Echocardiography Laboratory, he is involved in many clinical research projects as well. In addition, he is an avid programmer and is the author of a CasePro, a clinical data management system software.

Dr. Schwarz is a fellow of the American College of Cardiology and the American Heart Association. He is also a member of the American Society of Echocardiography.

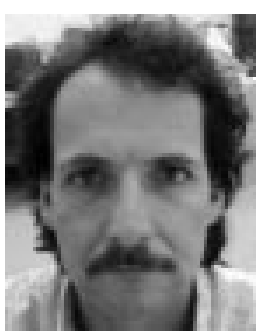

Daniel Phillips was born in Rochester, NY, in 1956. He received the B.S. degree in electrical engineering from the State University of New York at Buffalo in 1979. He received the M.S.E.E. degree in electrical engineering from the University of Rochester, Rochester, NY, in 1994 and is currently working toward the Ph.D. degree with a concentration in biomedical ultrasound.

From 1978 until 1992, he was employed as a Systems Engineer involved with automated test in the industrial sector, as well as medical data acquisition and analysis in both clinical and laboratory environments. His current research involves simulation and analysis of ultrasonic scattering in tissue.

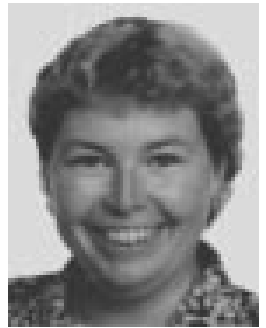

Sherry D. Steinmetz received the associate degree in applied science from the State University Agricultural and Technical College, Delhi, NY, in 1980, with a major in Veterinary Science Technology.

She has been working in the University of Rochester as a Laboratory Technician and Clinical Technician in radiology, nephrology, and most recently, cardiology. She is experienced in various surgical procedures in large and small animals as it pertains to echocardiographic protocols as well as angiographic protocols. She is also experienced in in-vitro echocardiographic studies.

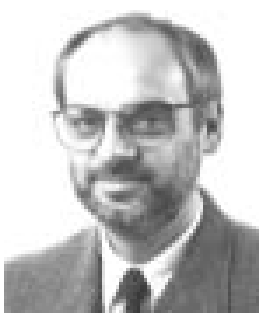

Reinhard Schlief was born in 1949 in Bochum, Germany. He graduated with a degree in physics in 1977. He received the M.D. degree from the Medical School of the Free University of Berlin Berlin, Germany.

He joined Schering AG Berlin, Berlin, Germany, in 1995 in the Division of Contrast Media Development. He has been Director of various clinical studies in ultrasound and magnetic resonance contrast media development since 1995 . He has been the Head of Department of Clinical Development Magnetic Resonance and Ultrasound Contrast Media since 1990. Dr. Schlief became a licensed physician in 1983. 